PROCEEDINGS OF THE

AMERICAN MATHEMATICAL SOCIETY

Volume 125, Number 12, December 1997, Pages 3659-3667

S 0002-9939(97)04199-3

\title{
MULTIPLE SOLUTIONS FOR A CLASS OF SEMILINEAR ELLIPTIC EQUATIONS
}

\author{
ZHIREN JIN
}

(Communicated by Jeffrey B. Rauch)

\begin{abstract}
We show that for a class of semilinear elliptic equations there are at least three nontrivial solutions. Existence of infinitely many solutions is also shown when the nonlinear term is odd. In our results, the nonlinear term can grow super-critically at infinity.
\end{abstract}

\section{INTRODUCTION AND RESULTS}

Let $\Omega$ be a $C^{2}$ bounded domain in $R^{n}, n \geq 3$. We consider the boundary value problem for a semilinear elliptic equation:

$$
\left\{\begin{array}{l}
-\Delta u=f(x, u, \lambda) \quad \text { on } \quad \Omega ; \\
u=0 \quad \text { on } \partial \Omega,
\end{array}\right.
$$

where $\Delta$ is the standard Laplace operator, $\lambda$ is a parameter close to 0 , the function $f(x, t, \lambda)$ is Hölder continuous for $(x, t) \in \bar{\Omega} \times R$ for each fixed $\lambda$.

A special case of $\left(P_{\lambda}\right)$ is the problem

$$
\left\{\begin{array}{l}
-\Delta u=\lambda|u|^{q-1} u+G^{\prime}(u) \quad \text { on } \quad \Omega \\
u=0 \quad \text { on } \partial \Omega
\end{array}\right.
$$

where $0<q<1, G(t)$ is a function on $t \in R$. When $G(t)=\frac{1}{p+1}|t|^{p+1}, 0<$ $q<1<p \leq(n+2) /(n-2)$, Ambrosetti, Brezis and Cerami showed in [2] that there is a number $\lambda_{*}>0$ such that for all $0<\lambda<\lambda_{*},(1.1)_{\lambda}$ has infinitely many solutions with negative energy. When $G(t)=\frac{1}{\alpha}|t|^{\alpha}+o\left(|s|^{\alpha}\right)$ near $t=0$ and $t=\infty$, $2<\alpha \leq \frac{2 n}{n-2}$, Ambrosetti, Azorero and Peral showed in [1] that there is a $\lambda^{*}>0$ such for all $0<\lambda<\lambda^{*},(1.1)_{\lambda}$ has at least three solutions with negative energy. Here we note in both cases the nonlinear term $G^{\prime}(u)$ grows at most as fast as $|u|^{(n+2) /(n-2)}$ at infinity.

In this note, we shall generalize above results to remove the restriction that $G^{\prime}(u)=O\left(|u|^{(n+2) /(n-2)}\right)$. We shall use the following assumptions.

$\left(f_{1}\right)$ There are constants $p>1, M>0$ and a function $c(\lambda)$ with $c(\lambda) \longrightarrow 0$ as $\lambda \longrightarrow 0$ such that

$$
|f(x, t, \lambda)| \leq c(\lambda)+M|t|^{p} \quad \text { for } \quad x \in \bar{\Omega}, \quad t \in R \quad \text { and } \quad 0<\lambda<1 .
$$

Received by the editors July 16, 1996.

1991 Mathematics Subject Classification. Primary 35J65, 35J25.

Key words and phrases. Multiple solutions, semilinear elliptic equations, sub- super-solutions, variational method, pseudo-gradient flow, local minimum of a functional.

(C)1997 American Mathematical Society 
$\left(f_{2}\right) \quad f(x, 0, \lambda)=0$ for $x \in \bar{\Omega}, 0<\lambda<1$.

$\left(f_{3}\right)$ For each fixed $\lambda$,

$$
\liminf _{t \rightarrow 0} \frac{f(x, t, \lambda)}{t}>\lambda_{2} \quad \text { uniformly for } \quad x \in \bar{\Omega}
$$

where $\lambda_{2}$ is the second eigenvalue of the Laplace operator with Dirichlet boundary conditions.

$\left(f_{4}\right) \quad$ There is a number $\eta>0$ such that $F(x, t, \lambda)=\int_{0}^{t} f(x, s, \lambda) d s>0$ for $x \in \bar{\Omega},-\eta \leq t \leq \eta, t \neq 0$ and $0<\lambda<1$.

$\left(f_{5}\right)$ For each fixed $\lambda$,

$$
\lim _{t \longrightarrow 0} \frac{f(x, t, \lambda)}{t}=\infty \quad \text { uniformly for } \quad x \in \bar{\Omega} .
$$

It is well known that a solution of $\left(P_{\lambda}\right)$ corresponds to a critical point of the functional

$$
I(u, \lambda)=\frac{1}{2} \int_{\Omega}|\nabla u|^{2} d x-\int_{\Omega} F(x, u, \lambda) d x, \quad u \in H_{0}^{1}(\Omega) .
$$

Our main results are

Theorem 1. Assume $\left(f_{1}\right)-\left(f_{4}\right)$. Then there is a number $\lambda_{0}>0$ such that for $0<\lambda<\lambda_{0},\left(P_{\lambda}\right)$ has at least three solutions satisfying $I(u, \lambda)<0$.

Theorem 2. Assume $\left(f_{1}\right),\left(f_{2}\right),\left(f_{5}\right)$ and $f(x, t, \lambda)$ is odd about $t$. Then there is a number $\lambda_{0}>0$ such that for $0<\lambda<\lambda_{0}$, $\left(P_{\lambda}\right)$ has infinitely many solutions satisfying $I(u, \lambda)<0$.

The idea of the proof is to truncate the nonlinear term $f(x, u, \lambda)$ by a pair of super- and sub- solutions so that the solutions to the new equation are solutions of $\left(P_{\lambda}\right)$. Then we prove Theorems 1 and 2 for the new equation.

From the proof, it will be clear that Theorems 1 and 2 hold if the Laplace operator in $\left(P_{\lambda}\right)$ is replaced by a more general second order linear elliptic operator in divergence form

$$
-\sum_{i, j=1}^{n} \partial_{j}\left(a_{i j}(x) \partial_{i} u\right)+\sum_{i=1}^{n} b_{i}(x) \partial_{i} u+c(x) u, \quad c(x) \geq 0 \quad \text { on } \quad \Omega .
$$

(Then $\lambda_{2}$ in $\left(f_{3}\right)$ is the second eigenvalue of the elliptic operator with Dirichlet boundary conditions.)

\section{THE PROOFS}

Let $\lambda_{1}$ be the first eigenvalue of the Laplace operator with Dirichlet boundary conditions. Let $\phi(x)$ be the positive eigenfunction of $\lambda_{1}$ with $\max \phi(x)=1$. That is,

$$
-\Delta \phi(x)=\lambda_{1} \phi(x) \quad \text { on } \quad \Omega, \quad \phi(x)=0 \quad \text { on } \quad \partial \Omega .
$$

First we show that $\left(P_{\lambda}\right)$ has a pair of super- sub- solutions with some special properties.

Lemma 1. There is a $\lambda_{0}>0$ such that for $0<\lambda<\lambda_{0}$, there are two $C^{2}$ functions $u_{+}(x)$ and $u_{-}(x)$ satisfying

1) $0<u_{+}(x)<\eta$ and $-\eta<u_{-}(x)<0$ on $\Omega$ (where $\eta$ is given in $\left(f_{4}\right)$ ); $u_{+}(x)=0$ and $u_{-}(x)=0$ on $\partial \Omega$; 
2) $\frac{\partial u_{+}}{\partial \mu}<0$, $\frac{\partial u_{-}}{\partial \mu}>0$ on $\partial \Omega$, where $\mu$ is the outer normal along $\partial \Omega$;

3) $-\Delta u_{+}(x) \geq M\left\|u_{+}\right\|_{\infty}^{p}+c(\lambda)+\lambda,-\Delta u_{-}(x) \leq-M\left\|u_{-}\right\|_{\infty}^{p}-c(\lambda)-\lambda$ on $\Omega$.

Remark 1. $\left(f_{1}\right)$ implies that $u_{+}(x), u_{-}(x)$ are a pair of super- sub- solutions of $\left(P_{\lambda}\right)$.

Proof. Here we construct $u_{+}(x)$ only. $u_{-}(x)$ can be constructed similarly. The construction is the same as that given in the proof of Lemma 3.1 on page 524 in $[2]$. For reader's convenience we sketch it here.

Let $e(x)$ satisfy $-\Delta e(x)=1$ on $\Omega, e(x)=0$ on $\partial \Omega$. Since $p>1$ and $c(\lambda) \longrightarrow 0$ as $\lambda \longrightarrow 0$, there is a $\lambda_{0}>0$ such that for $0<\lambda<\lambda_{0}$, there is a number $M_{1}=M_{1}(\lambda)$ satisfying $M_{1}\|e\|_{\infty}<\eta$ and

$$
M_{1}>M\left(\|e\|_{\infty} M_{1}\right)^{p}+c(\lambda)+\lambda .
$$

Then

$$
-\Delta\left(M_{1} e(x)\right)=M_{1}>M\left(\|e\|_{\infty} M_{1}\right)^{p}+c(\lambda)+\lambda .
$$

Thus we can set $u_{+}(x)=M_{1} e(x)$. The property that $\frac{\partial u_{+}}{\partial \mu}<0$ on $\partial \Omega$ follows from the definition of $e(x)$ and the Hopf boundary maximum principle (see [6]).

We now use $u_{+}(x)$ and $u_{-}(x)$ to truncate the nonlinear term $f(x, u, \lambda)$ in $\left(P_{\lambda}\right)$. Let $\psi(t)$ be a $C^{2}$ cut off function satisfying: $\psi(t)=0$ if $t<0, \psi(t)=1$ if $t>1$, $0 \leq \psi(t) \leq 1$. Let $\delta(\lambda)$ be a small positive constant to be determined later. We set

$$
\begin{array}{r}
h_{+}(x, t, \lambda)=f\left(x,\left\|u_{+}\right\|_{\infty}, \lambda\right)\left(1-\psi\left(\frac{t-\left\|u_{+}\right\|_{\infty}}{\delta(\lambda)}\right)\right) \\
+\psi\left(\frac{t-\left\|u_{+}\right\|_{\infty}}{\delta(\lambda)}\right)\left(M\left\|u_{+}\right\|_{\infty}^{p}+c(\lambda)+\frac{1}{2} \lambda\right) ; \\
h_{-}(x, t, \lambda)=f\left(x,-\left\|u_{-}\right\|_{\infty}, \lambda\right)\left(1-\psi\left(\frac{-\left\|u_{-}\right\|_{\infty}-t}{\delta(\lambda)}\right)\right) \\
-\psi\left(\frac{-\left\|u_{-}\right\|_{\infty}-t}{\delta(\lambda)}\right)\left(M\left\|u_{-}\right\|_{\infty}^{p}+c(\lambda)+\frac{1}{2} \lambda\right) ;
\end{array}
$$

and

$$
\begin{gathered}
g_{+}(x, t, \lambda)=\left\{\begin{array}{l}
M\left\|u_{+}\right\|_{\infty}^{p}+c(\lambda)+\frac{1}{2} \lambda \quad \text { if } \quad t>\left\|u_{+}\right\|_{\infty}+\delta(\lambda) ; \\
h_{+}(x, t, \lambda) \quad \text { if }\left\|u_{+}\right\|_{\infty} \leq t \leq\left\|u_{+}\right\|_{\infty}+\delta(\lambda) ; \\
f(x, t, \lambda) \quad \text { if } 0<t<\left\|u_{+}\right\|_{\infty} ; \\
0 \quad \text { if } t \leq 0 .
\end{array}\right. \\
g_{-}(x, t, \lambda)=\left\{\begin{array}{l}
0 \quad \text { if } t \geq 0 ; \\
f(x, t, \lambda) \quad \text { if } \quad-\left\|u_{-}\right\|_{\infty}<t<0 ; \\
h_{-}(x, t, \lambda) \quad \text { if }-\left\|u_{-}\right\|_{\infty}-\delta(\lambda) \leq t \leq-\left\|u_{-}\right\|_{\infty} ; \\
-M\left\|u_{-}\right\|_{\infty}^{p}-c(\lambda)-\frac{1}{2} \lambda \quad \text { if } t<-\left\|u_{+}\right\|_{\infty}-\delta(\lambda) .
\end{array}\right.
\end{gathered}
$$

Then we have the following properties for $g_{+}(x, t, \lambda)$ and $g_{-}(x, t, \lambda)$ :

$\left(g_{1}\right) \quad g_{+}(x, t, \lambda)$ and $g_{-}(x, t, \lambda)$ are bounded, Hölder continuous functions on $(x, t)$ for each fixed $0<\lambda<\lambda_{0}$; 
$\left(g_{2}\right) \quad g_{+}(x, t, \lambda)=f(x, t, \lambda)$ on $x \in \Omega$ if $0<t \leq\left\|u_{+}\right\|_{\infty}$;

(g) $g_{-}(x, t, \lambda)=f(x, t, \lambda)$ on $x \in \Omega$ if $-\left\|u_{-}\right\|_{\infty} \leq t<0$;

$\left(g_{4}\right) \quad g_{+}(x, t, \lambda)<M\left\|u_{+}\right\|_{\infty}^{p}+c(\lambda)+\lambda$ for $t>0, x \in \Omega$

$\left(g_{5}\right) \quad g_{-}(x, t, \lambda)>-M\left\|u_{-}\right\|_{\infty}^{p}-c(\lambda)-\lambda$ for $t<0, x \in \Omega$.

Indeed $\left(g_{1}\right)-\left(g_{3}\right)$ follow directly from the definitions. $\left(g_{4}\right)$ and $\left(g_{5}\right)$ follow from the definitions and $\left(f_{1}\right)$. We also claim that if we choose $\delta(\lambda)$ small enough, we have

$\left(g_{6}\right) \quad \int_{0}^{t} g_{+}(x, s, \lambda) d s \geq 0$ for all $t>0, x \in \Omega$.

$\left(g_{7}\right) \quad \int_{0}^{t} g_{-}(x, s, \lambda) d s \geq 0$ for all $t<0, x \in \Omega$.

In fact, $\left(g_{6}\right)$ and $\left(g_{7}\right)$ follows from $\left(f_{4}\right)$. For example, for $\left(g_{6}\right)$, since $\left\|u_{+}\right\|_{\infty}<\eta$, $\left(f_{4}\right)$ implies that $F\left(x,\left\|u_{+}\right\|_{\infty}, \lambda\right) \geq c_{1}>0$ on $x \in \bar{\Omega}$ for some constant $c_{1}$. Then we can choose a small $\delta(\lambda)>0$ such that for $\left\|u_{+}\right\|_{\infty} \leq t \leq\left\|u_{+}\right\|_{\infty}+\delta(\lambda)$,

$$
\begin{gathered}
\int_{0}^{t} g_{+}(x, s, \lambda) d s=\int_{0}^{\left\|u_{+}\right\|_{\infty}} g_{+}(x, s, \lambda) d s+\int_{\left\|u_{+}\right\|_{\infty}}^{t} g_{+}(x, s, \lambda) d s \\
=F\left(x,\left\|u_{+}\right\|_{\infty}, \lambda\right)+\int_{\left\|u_{+}\right\|_{\infty}}^{t} g_{+}(x, s, \lambda) d s \geq c_{1}-\delta(\lambda)\left(M\left\|u_{+}\right\|_{\infty}^{p}+c(\lambda)+\lambda\right)>0
\end{gathered}
$$

where we have used $\left(g_{2}\right)$ and $\left(g_{4}\right)$. For the remaining case of $t$, it is easy to see $\left(g_{6}\right)$.

Now we define $f^{*}(x, t, \lambda)=g_{+}(x, t, \lambda)+g_{-}(x, t, \lambda)$ and consider the boundary value problem:

$$
\left\{\begin{array}{l}
-\Delta u=f^{*}(x, u, \lambda) \quad \text { on } \Omega ; \\
u=0 \quad \text { on } \partial \Omega .
\end{array}\right.
$$

From $\left(g_{1}\right)-\left(g_{7}\right)$, we have the following properties:

$\left(f_{1}^{*}\right) f^{*}(x, t, \lambda)$ is bounded for each fixed $0<\lambda<\lambda_{0}$;

$\left(f_{2}^{*}\right) u_{+}(x)$ is a super solution, $u_{-}(x)$ is a sub-solution of $\left(P_{\lambda}^{*}\right)$;

$\left(f_{3}^{*}\right) f^{*}(x, t, \lambda)=f(x, t, \lambda)$ for $x \in \Omega,-\left\|u_{-}\right\|_{\infty} \leq t \leq\left\|u_{+}\right\|_{\infty}$, and $0<\lambda<\lambda_{0}$;

$\left(f_{4}^{*}\right) F^{*}(x, t, \lambda)=\int_{0}^{t} f^{*}(x, s, \lambda) \geq 0$ for all $x \in \Omega, t \in R, 0<\lambda<\lambda_{0}$;

$\left(f_{5}^{*}\right)$ If $f(x, t, \lambda)$ is odd in $t$, we can choose $u_{+}(x)=-u_{-}(x)$. Thus $f^{*}(x, t, \lambda)$ is also odd in $t$.

The next lemma shows the reason that we introduce the problem $\left(P_{\lambda}^{*}\right)$. That is, we only need to prove Theorems 1 and 2 for $\left(P_{\lambda}^{*}\right)$ instead of $\left(P_{\lambda}\right)$.

Lemma 2. Every $C^{2}$ solution $u(x)$ of $\left(P_{\lambda}^{*}\right)$ is a solution of $\left(P_{\lambda}\right)$.

Proof. Let $u(x)$ be a solution of $\left(P_{\lambda}^{*}\right)$. Then on $\Omega_{1}=\left\{x \mid u(x)>u_{+}(x)\right\}$, from $\left(g_{4}\right)$ and the definition of $u_{+}$, on $\Omega_{1}$, we have

$$
-\Delta u=f^{*}(x, u, \lambda)=g_{+}(x, u(x), \lambda) \leq M\left\|u_{+}\right\|_{\infty}^{p}+c(\lambda)+\lambda \leq-\Delta u_{+}(x) .
$$

Thus $-\Delta\left(u-u_{+}\right)(x) \leq 0$ on $\Omega_{1}$ and $u(x)-u_{+}(x)$ cannot achieve a maximum inside $\Omega_{1}$. But $u(x)=u_{+}(x)$ on $\partial \Omega$ and $\partial \Omega_{1}$. Thus $\Omega_{1}$ must be empty. That is $u(x) \leq u_{+}(x)$ on $\Omega$. Similarly we can prove that $u_{-}(x) \leq u(x)$ on $\Omega$. Now $\left(g_{2}\right)$ and $\left(g_{3}\right)$ imply $f^{*}(x, u(x), \lambda)=f(x, u(x), \lambda)$ for $x \in \Omega$ and $u(x)$ solves $\left(P_{\lambda}\right)$.

In the rest of the paper, we shall prove Theorems 1 and 2 for the problem $\left(P_{\lambda}^{*}\right)$. We consider the functional

$$
I^{*}(u, \lambda)=\frac{1}{2} \int_{\Omega}|\nabla u|^{2} d x-\int_{\Omega} F^{*}(x, u, \lambda) d x, \quad u \in H_{0}^{1}(\Omega),
$$


where $F^{*}(x, t, \lambda)=\int_{0}^{t} f^{*}(x, s, \lambda)$. We first follow the idea used in the Lemma 4.1 on page 533 in [2] to show that $I^{*}(u, \lambda)$ has two local minimums with negative energy on $H_{0}^{1}(\Omega)$.

Lemma 3. 1) There is a positive function $v_{+}(x)$ which is a local minimum of $I^{*}(u, \lambda)$ in $H_{0}^{1}(\Omega)$ and $I^{*}\left(v_{+}, \lambda\right)<0$.

2) There is a negative function $v_{-}(x)$ which is a local minimum of $I^{*}(u, \lambda)$ in $H_{0}^{1}(\Omega)$ and $I^{*}\left(v_{-}, \lambda\right)<0$.

Proof. 1): We consider the functional

$$
I_{+}(u, \lambda)=\frac{1}{2} \int_{\Omega}|\nabla u|^{2} d x-\int_{\Omega} F_{+}(x, u, \lambda) d x, \quad u \in H_{0}^{1}(\Omega),
$$

where $F_{+}(x, t, \lambda)=\int_{0}^{t} g_{+}(x, s, \lambda)$. It is easy to see that $I^{*}(u, \lambda)=I_{+}(u, \lambda)$ for all $u \in H_{0}^{1}(\Omega)$ with $u(x) \geq 0$ on $\Omega$.

Since $g_{+}(x, t, \lambda)$ is bounded, $I_{+}(u, \lambda)$ has a global minimum $v_{+}(x)$ on $H_{0}^{1}(\Omega)$. Then

$$
-\Delta v_{+}=g_{+}\left(x, v_{+}, \lambda\right) \quad \text { on } \quad \Omega, \quad v_{+}=0 \quad \text { on } \quad \partial \Omega .
$$

As we did in the proof of Lemma 2, we have

$$
v_{+}(x)<u_{+}(x) \text { on } \Omega .
$$

Furthermore $\left(g_{2}\right),\left(g_{4}\right)$ and the Hopf's boundary maximum principle imply that for the outer normal $\mu$ along $\partial \Omega$,

$$
\frac{\partial\left(v_{+}-u_{+}\right)}{\partial \mu}>0 \quad \text { on } \quad \partial \Omega .
$$

On the other hand, the definition of $g_{+}(x, t, \lambda)$ and maximum principle imply that $v_{+}(x) \geq 0$ on $\Omega$. If $v_{+} \neq 0$ (which follows from $I\left(v_{+}, \lambda\right)<0$ ), then $\left(g_{2}\right),\left(f_{3}\right)$ and the Hopf's boundary maximum principle imply that $v_{+}>0$ and

$$
\frac{\partial v_{+}}{\partial \mu}<0 \quad \text { on } \quad \partial \Omega
$$

Therefore (2.4), (2.5) and (2.6) imply that $v_{+}$is a local minimum of $I_{+}(u, \lambda)$ in $C^{1}$ topology. Since $v_{+}>0$ on $\Omega,(2.6)$ and the definition of $I_{+}(u, \lambda)$ imply that $v_{+}$is also a local minimum of $I^{*}(u, \lambda)$ in $C^{1}$ topology. Then $v_{+}(x)$ is a local minimum of $I^{*}(u, \lambda)$ in $H_{0}^{1}(\Omega)$ by [4]. Now it still remains to show that $I^{*}\left(v_{+}, \lambda\right)<0$. We only need to show $I_{+}\left(v_{+}, \lambda\right)<0$. Indeed $\left(g_{2}\right)$ and $\left(f_{3}\right)$ imply that for $t>0$ small, $g_{+}(x, t, \lambda)=f(x, t, \lambda)>\frac{1}{2}\left(\lambda_{1}+\lambda_{2}\right) t$ for $x \in \Omega$. Then for $s$ small and the first eigenfunction $\phi(x)$ defined in (2.1),

$$
\begin{gathered}
I_{+}(s \phi, \lambda)=\frac{s^{2}}{2} \int_{\Omega}|\nabla \phi|^{2} d x-\int_{\Omega} F_{+}(x, s \phi(x), \lambda) d x \\
\leq \frac{s^{2} \lambda_{1}}{2} \int_{\Omega} \phi^{2} d x-\frac{\left(\lambda_{1}+\lambda_{2}\right) s^{2}}{4} \int_{\Omega} \phi^{2} d x<0 .
\end{gathered}
$$

Thus $I_{+}\left(v_{+}, \lambda\right)<0$ since $v_{+}$is a global minimum.

$2)$ : The argument here is similar to that in 1 ). Now we consider the functional

$$
I_{-}(u, \lambda)=\frac{1}{2} \int_{\Omega}|\nabla u|^{2} d x-\int_{\Omega} F_{-}(x, u, \lambda) d x, \quad u \in H_{0}^{1}(\Omega) .
$$


where $F_{-}(x, t, \lambda)=\int_{0}^{t} g_{-}(x, s, \lambda)$. It is easy to see that $I^{*}(u, \lambda)=I_{-}(u, \lambda)$ for all $u \in H_{0}^{1}(\Omega)$ with $u(x) \leq 0$ on $\Omega$. Then we can show that $I_{-}(u, \lambda)$ has a global minimum $v_{-}(x)<0$ which is what we want. We leave the detail to the reader.

Now $I^{*}(u, \lambda)$ has two nontrivial critical points $v_{+}$and $v_{-}$. Thus $\left(P_{\lambda}^{*}\right)$ and $\left(P_{\lambda}\right)$ has two solutions $v_{+}$and $v_{-}$. The third critical point will be constructed by the Mountain-Pass critical level of $I^{*}(u, \lambda)$ with base points $v_{+}$and $v_{-}$. Before we get into the construction of the third critical point, we state some properties for the functionals $I_{+}(u, \lambda)$ and $I_{-}(u, \lambda)$ defined in the proof of Lemma 3. First from the definitions and maximum principles, we have

Lemma 4. Each critical point of $I_{+}(u, \lambda)$ or $I_{-}(u, \lambda)$ is a solution of $\left(P_{\lambda}^{*}\right)$.

Since $f^{*}(x, u, \lambda), g_{+}(x, u, \lambda)$ and $g_{-}(x, u, \lambda)$ are all bounded on $\Omega \times R$ for each $\lambda$, we have

Lemma 5. $I^{*}(u, \lambda), I_{+}(u, \lambda)$ and $I_{-}(u, \lambda)$ are bounded from below and satisfy Palais-Smale condition on $H_{0}^{1}(\Omega)$.

Now we are ready to prove Theorem 1.

Proof of Theorem 1. We shall use the same notations as those in Lemmas 1-5.

Step 1 . There is a $t_{+}(\lambda)>0$, such that for $0<s<t_{+}(\lambda)$,

$$
I_{+}(s \phi(x), \lambda)<0
$$

where $\phi(x)$ is defined in $(2.1)$.

This is exactly what we computed in (2.7).

Step 2. Similar to Step 1 , there is a $t_{-}(\lambda)<0$, such that for $t_{-}(\lambda)<s<0$,

$$
I_{+}(s \phi(x), \lambda)<0 .
$$

Step 3. Let $W_{+}(u)$ be the pseudo-gradient vector field of $I_{+}(u, \lambda)$. We consider the pseudo-gradient flow

$$
\frac{d u}{d t}=-W_{+}(u) \quad \text { for } \quad t>0 ; \quad u(0)=s \phi(x),
$$

where $0<s<t_{+}(\lambda)$ is defined in Step 1. From the properties of pseudo-gradient vector field, we know that $u(t)$ exists for all $t>0$. We claim that there is a sequence of $t_{k} \longrightarrow \infty$, such that $u\left(t_{k}\right) \longrightarrow w_{+}$in $H_{0}^{+}(\Omega)$ and $w_{+}$is a critical point of $I_{+}(u, \lambda)$ with $I_{+}\left(w_{+}, \lambda\right)<0$.

The claim will follow from the fact that $\left\|I_{+}^{\prime}(u(t), \lambda)\right\|$ is not bounded away from zero as $t \longrightarrow \infty$. Indeed if $\left\|I_{+}^{\prime}(u(t), \lambda)\right\| \geq c>0$ for $t$ large, we have that for $t$ large

$$
\frac{d}{d t} I_{+}(u(t), \lambda)=\left\langle I_{+}^{\prime}(u(t), \lambda), \frac{d u}{d t}\right\rangle=\left\langle I_{+}^{\prime}(u(t), \lambda),-W_{+}(u(t))\right\rangle \leq-c_{2}<0 .
$$

Thus $I_{+}(u(t), \lambda) \longrightarrow-\infty$ as $t \longrightarrow \infty$. This contradicts to the fact that $I_{+}(u, \lambda)$ is bounded from below on $H_{0}^{1}(\Omega)$. Thus there is a sequence of $t_{k} \longrightarrow \infty$, such that $I_{+}^{\prime}\left(u\left(t_{k}\right), \lambda\right)$ approaches to zero. But it is also clear that $I_{+}(u, \lambda) \longrightarrow \infty$ as $\|u\| \longrightarrow$ $\infty$ (since $g_{+}(x, u, \lambda)$ is bounded). Thus $u\left(t_{k}\right)$ is bounded since $I_{+}\left(u\left(t_{k}\right), \lambda\right) \leq$ $I_{+}(u(0), \lambda)=I_{+}(s \phi(x), \lambda)<0$. Then the Palais-Smale condition and Lemma 5 imply that $u\left(t_{k}\right)$ has a convergence subsequence. We still denote the subsequence by $u\left(t_{k}\right)$. Then $u\left(t_{k}\right) \longrightarrow w_{+}$in $H_{0}^{1}(\Omega)$ for some $w_{+}, I_{+}^{\prime}\left(w_{+}, \lambda\right)=\lim I_{+}^{\prime}\left(u\left(t_{k}\right), \lambda\right)=0$ and $I_{+}\left(w_{+}, \lambda\right)=\lim I_{+}\left(u\left(t_{k}\right), \lambda\right) \leq I_{+}(u(0), \lambda)=I_{+}(s \phi(x), \lambda)<0$. 
Step 4 . Let $W_{-}(u)$ be the pseudo-gradient vector field of $I_{-}(u, \lambda)$. We consider the pseudo-gradient flow

$$
\frac{d u}{d t}=-W_{-}(u) \quad \text { for } \quad t>0 ; \quad u(0)=s \phi(x),
$$

where $t_{-}(\lambda)<s<0$ is defined in Step 2. Then there is a sequence of $t_{j} \longrightarrow \infty$, such that $u\left(t_{j}\right) \longrightarrow w_{-}$in $H_{0}^{1}(\Omega)$ and $w_{-}$is a critical point of $I_{-}(u, \lambda)$ with $I_{-}\left(w_{-}, \lambda\right)$ $<0$. The argument here is similar to that in Step 3.

Step 5. Let $\phi_{2}(x)$ be an eigenfunction corresponding to the second eigenvalue $\lambda_{2}$ of the Laplace operator with Dirichlet boundary conditions. Let $V$ be the two dimensional subspace of $H_{0}^{1}(\Omega)$ spanned by $\phi(x)$ and $\phi_{2}(x)$. Then we claim that for each fixed $\lambda$, there is a $\delta>0$ such that if $0<\epsilon<\delta, u \in V$ and $\|u\|=\epsilon$, we have

$$
I^{*}(u, \lambda)<0 .
$$

First it is easy to see that for $u \in V$,

$$
\int_{\Omega}|\nabla u|^{2} d x \leq \lambda_{2} \int_{\Omega} u^{2} d x
$$

From $\left(f_{3}\right)$, there are $t_{3}>0\left(t_{3}<\min \left\{\left\|u_{+}\right\|_{\infty},\left\|u_{-}\right\|_{\infty}\right\}\right)$ and $\delta_{1}>0$ such that $F(x, t, \lambda)=\int_{0}^{t} f(x, s, \lambda) d s \geq \frac{1}{2}\left(\lambda_{2}+\delta_{1}\right) t^{2}$ for $|t|<t_{3}$. Now we take a $\delta>0$, such that if $0<\epsilon<\delta, u \in V,\|u\|=\epsilon$, we have $|u(x)|<t_{3}$ for $x \in \Omega, u_{-}(x)<u(x)<$ $u^{+}(x)$; then from $\left(g_{2}\right),\left(g_{3}\right)$ we have

$$
\begin{aligned}
I^{*}(u, \lambda) & =\frac{1}{2} \int_{\Omega}|\nabla u|^{2} d x-\int_{\Omega} F^{*}(x, u, \lambda) d x \\
& =\frac{1}{2} \int_{\Omega}|\nabla u|^{2} d x-\int_{\Omega} F(x, u, \lambda) d x \leq \frac{1}{2} \epsilon^{2}-\frac{1}{2}\left(\lambda_{2}+\delta_{1}\right) \int_{\Omega} u^{2} d x \\
& \leq \frac{1}{2} \epsilon^{2}-\frac{\lambda_{2}+\delta_{1}}{2 \lambda_{2}} \epsilon^{2}<0 .
\end{aligned}
$$

Here we have used (2.11).

Step 6. For $v_{+}, v_{-}$defined in Lemma $3, w_{+}, w_{-}$defined in Steps 3 and 4 , if $v_{+} \neq w_{+}$or $v_{-} \neq w_{-}$, the conclusion of Theorem 1 follows immediately. Thus we may assume $v_{+}=w_{+}$and $v_{-}=w_{-}$.

Let us define $b(\lambda)$, the Mountain-Pass critical level of $I^{*}(u, \lambda)$ with base points $v_{+}$and $v_{-}$:

$$
b(\lambda)=\inf _{h \in A} \max \left\{I^{*}(h(t), \lambda) \mid t \in[0,1]\right\}
$$

where $A=\left\{h \in C^{0}\left([0,1], H_{0}^{1}(\Omega)\right) \mid h(0)=v_{+}, h(1)=v_{-}\right\}$.

Since $I^{*}(u, \lambda)$ satisfies the Palais-Smale condition and $v_{+}$and $v_{-}$are local minima of $I^{*}(u, \lambda)$ in $H_{0}^{1}(\Omega), b(\lambda)$ is a critical value of $I^{*}(u, \lambda)$ (see [3] and [5]). To finish the proof we only need to demonstrate that there is a path $h(t) \in A$, such that

$$
\max \left\{I^{*}(h(t), \lambda) \mid t \in[0,1]\right\}<0 .
$$

Since $I^{*}\left(v_{+}, \lambda\right)<0$, there is a neighborhood $D_{+}$of $v_{+}$such that $I^{*}(u, \lambda)<c_{5}<0$ for $u \in D_{+} \cdot v_{+}=w_{+}$and $u\left(t_{k}\right) \longrightarrow w_{+}$as $k \longrightarrow \infty(u(t)$ is the pseudo-gradient flow defined in Step 3) imply that $u\left(t_{k}\right) \in D_{+}$for some $u\left(t_{k}\right)$. We also know that 
$I_{+}(u(t), \lambda) \leq I_{+}(u(0), \lambda)=I_{+}(s \phi(x), \lambda)<0$ for $0<t<t_{k}$. Then from $\left(f_{4}^{*}\right)$ we have (here $\left.u^{+}(t)(x)=\max \{u(t)(x), 0\}, u^{-}(t)(x)=\min \{u(t)(x), 0\}\right)$

$$
\begin{gathered}
\int_{\Omega} F^{*}(x, u(t), \lambda) d x=\int_{\Omega} F^{*}\left(x, u^{+}(t), \lambda\right) d x+\int_{\Omega} F^{*}\left(x, u^{-}(t), \lambda\right) d x \\
\geq \int_{\Omega} F^{*}\left(x, u^{+}(t), \lambda\right) d x=\int_{\Omega} F_{+}(x, u(t), \lambda) d x .
\end{gathered}
$$

Then for $0<t<t_{k}$,

$$
\begin{gathered}
I^{*}(u(t), \lambda)=\frac{1}{2} \int_{\Omega}|\nabla u(t)|^{2} d x-\int_{\Omega} F^{*}(x, u(t), \lambda) d x \\
\leq \frac{1}{2} \int_{\Omega}|\nabla u(t)|^{2} d x-\int_{\Omega} F_{+}(x, u(t), \lambda) d x=I_{+}(u(t), \lambda)<I_{+}(s \phi, \lambda)<0 .
\end{gathered}
$$

Hence we can connect $v_{+}$to $s \phi$ by a path $h_{1}(t)$ which is from $v_{+}$to $u\left(t_{k}\right) \in D_{+}$ (any continuous curve) and from $u\left(t_{k}\right)$ to $s \phi$ (by the pseudo-gradient flow $u(t)$ ). It is clear that $\max I\left(h_{1}(t)\right)<0$. Similarly we can connect $v_{-}$to $-s \phi$ by a path $h_{2}$ such that $\max I\left(h_{2}\right)<0$. Now when $s$ is small, a path $h_{3}$ is provided by Step 5 such that $\max I\left(h_{3}\right)<0$. Finally the path $h(t)$ in $(2.12)$ can be obtained by the connecting paths $h_{1}, h_{3}$ and $h_{2}$.

Remark 2. It is clear from the proof of Lemma 2 that for all critical points $v$ of $I^{*}(u, \lambda)$, we have $I^{*}(v, \lambda)=I(v, \lambda)$.

Proof of Theorem 2. As we remarked before, when $f(x, t, \lambda)$ is odd in $t$, we can choose $f^{*}(x, t, \lambda)$ so that $f^{*}(x, t, \lambda)$ is also odd. And from Lemma 2 we only need to prove Theorem 2 for the functional $I^{*}(u, \lambda)$. The proof goes exactly like that of Theorem 2.5 in [2] since all of the following ingredients are in place:

1) there is a $r>0$ such that $I^{*}(u, \lambda)>0$ for all $\|u\| \geq r$.

2) $I^{*}(u, \lambda)$ is bounded from below on $H_{0}^{1}(\Omega)$.

3) $I^{*}(u, \lambda)$ satisfies the Palais-Smale condition.

4) Let $H_{k}$ be the linear subspace of $H_{0}^{1}(\Omega)$ spanned by the first $k$ eigenfunctions of the Laplace operator with Dirichlet boundary conditions, then for each $\lambda$, when $\epsilon$ is small

$$
I^{*}(u, \lambda)<0 \quad \text { for } \quad u \in H_{k},\|u\|=\epsilon .
$$

5) $I^{*}(u, \lambda)$ is even.

The properties 1)-3) follows from $\left(f_{1}^{*}\right)$. The property 4) follows from $\left(f_{3}^{*}\right)$ and $\left.\left(f_{5}\right) .5\right)$ follows from the fact that now $f^{*}(x, t, \lambda)$ is odd.

\section{BIBLIOGRAPHY}

1. Ambrosetti, A., Azorero, J.G. \& Peral, I., Multiplicity results for some nonlinear elliptic equations, J. Funct. Anal. 137 (1996), 219-242. MR 97b:35059

2. Ambrosetti, A., Brezis, H. \& Cerami, G., Combined effects of concave and convex nonlinearities in some elliptic problems, J. Funct. Anal. 122 (1994), 519-543. MR 95g:35059

3. Ambrosetti, A. \& Rabinowitz, P.H., Dual variational methods in critical point theory and applications, J. Funct. Anal. 14 (1973), 349-381. MR 51:6412

4. Brezis, H. \& Nirenberg, L., $H^{1}$ versus $C^{1}$ local minimizers, C.R. Acad. Sci. Paris 317 (1993), 465-472. MR 94g:49044 
5. Ghoussoub, N. \& Preiss, D., A general mountain pass principle for locating and classifying critical points, Ann. Inst. H. Poincaré Anal. Non Linéaire 6 (1989), 321-330. MR 91a:58043

6. Gilbarg, D. \& Trüdinger, N.S., Elliptic partial differential equations of second order, second edition, Springer-verlag (1983). MR 86c:35035

Department of Mathematics and Statistics, Wichita State University, Wichita, KANSAS 67260

E-mail address: zhiren@cs.twsu.edu 